\title{
Perspectives on novel therapeutic strategies for right heart failure in pulmonary arterial hypertension: lessons from the left heart
}

\author{
M.L. Handoko*,\#, F.S. de Man\#, C.P. Allaart, W.J. Paulus*, \\ N. Westerhof*,\# and A. Vonk-Noordegraaf*,\#
}

ABSTRACT: Right heart function is the main determinant of prognosis in pulmonary arterial hypertension (PAH). At present, no treatments are currently available that directly target the right ventricle, as we will demonstrate in this article.

Meta-analysis of clinical trials in PAH revealed that current PAH medication seems to have limited cardiac-specific effects when analysed by the pump-function graph. Driven by the hypothesis that "left" and right heart failure might share important underlying pathophysiological mechanisms, we evaluated the clinical potential of left heart failure (LHF) therapies for PAH, based on currently available literature.

As in LHF, the sympathetic nervous system and the renin-angiotension-aldosterone system are highly activated in PAH. From LHF we know that intervening in this process, e.g. by angiotensinconverting enzyme inhibition or $\beta$-blockade, is beneficial in the long run. Therefore, these medications could be also beneficial in PAH. Furthermore, the incidence of sudden cardiac death in PAH could be reduced by implantable cardioverter-defibrillators. Finally, pilot studies have demonstrated that interventricular dyssynchrony, present at end-stage $\mathrm{PAH}$, responded favourably to cardiac resynchronisation therapy as well.

In conclusion, therapies for LHF might be relevant for PAH. However, before they can be implemented in PAH management, safety and efficacy should be evaluated first in well-designed clinical trials.

KEYWORDS: Adrenergic $\beta$-antagonists, artificial cardiac pacing, implantable defibrillators, pulmonary heart disease, rennin-angiotensin system, right ventricular dysfunction

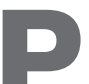
ulmonary arterial hypertension (PAH) is characterised by excessive pulmonary vascular remodelling, resulting in a marked increase in right ventricular (RV) afterload. The thin-walled, crescent-shaped right ventricle in the normal situation needs to remodel to a thick-walled, more spherical-shaped high-pressure pump to overcome the often fourfold increase in pressure in the case of PAH. Eventually, the right ventricle is not able to cope with the increase in load and right heart failure develops [1, 2]. Despite the successful introduction of several new pulmonary-selective vasodilating therapies in the last $10 \mathrm{yrs}$, the prognosis of PAH patients remains poor [3, 4].

The relationship between RV afterload (mainly determined by pulmonary vascular resistance
(PVR) and pulmonary arterial compliance [5]) and RV dysfunction is not straightforward. Patients with systemic sclerosis associated-PAH (relatively low load/low pressure) have a worse prognosis compared with patients with idiopathic $\mathrm{PAH}$, whereas patients with $\mathrm{PAH}$ associated with congenital heart disease (high load/high pressure) have a relatively good prognosis [6]. Also in PAH, mean pulmonary artery pressure $\left(\bar{P}_{\text {pa }}\right)$ and PVR are of limited prognostic value, while the strongest predictors of survival are reflections of RV (mal)adaptation to its increased load (cardiac index, right atrial pressure, tricuspid annular plane systolic excursion and N-terminal pro-brain natriuretic plasma levels) (fig. 1) [8-10]. Thus, it is not the load per se, but the failing right ventricle itself that leads to death.

\section{AFFILIATIONS}

Depts of *Physiology,

"Pulmonology, and

- Cardiology, VU University Medical Center/Institute for Cardiovascular Research, Amsterdam, The Netherlands.

CORRESPONDENCE

A. Vonk-Noordegraaf Dept of Pulmonology VU University Medical Center; Boelelaan 1117 1081 HV Amsterdam The Netherlands E-mail: a.vonk@vumc.nl

Received: Nov 232009 Accepted after revision: Dec 192009

PROVENANCE

Submitted article, peer reviewed.

European Respiratory Review Print ISSN 0905-9180 Online ISSN 1600-0617 


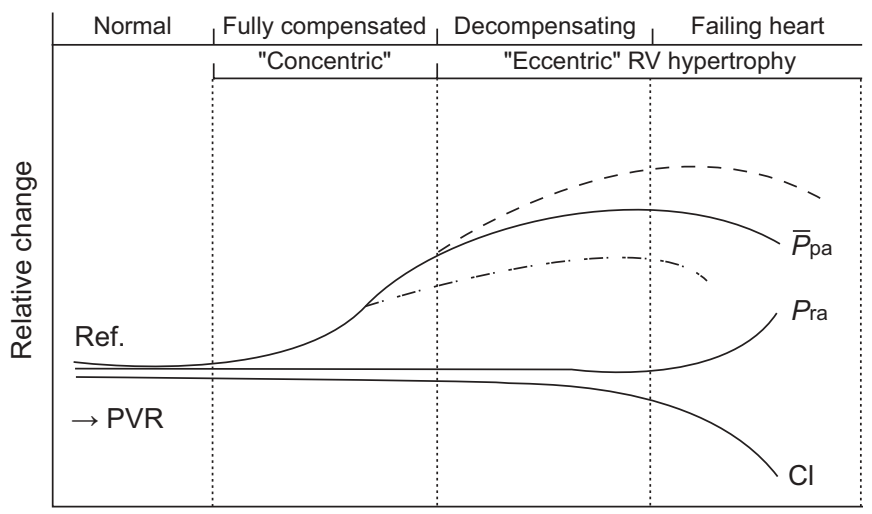

Progression of PAH

FIGURE 1. Haemodynamic changes during the progression of pulmonary arterial hypertension $(\mathrm{PAH})$. The continuous rise in pulmonary vascular resistance (PVR) during the progression of PAH is initially compensated by concentric remodelling of the right ventricle (RV). Right atrial pressure (Pra) remains normal and there is a steep increase in mean pulmonary artery pressure $(\bar{P} p a)$ as cardiac index $(\mathrm{Cl})$ at rest is preserved. In the next stage, the RV is not able to fully compensate for the further increase of PVR and starts to decompensate; eccentric RV remodelling is observed. There is a modest rise in $\bar{P}$ pa as $\mathrm{Cl}$ also starts to fall. At this stage Pra remains at near normal levels. In the final stage of overt right heart failure there is a severe drop in $\mathrm{Cl}$, a steep rise in Pra and, even though PVR still increases, $\bar{P}_{\text {pa }}$ drops due to the low output state. Changes in RV function fit to the different disease stages in PAH and explain the prognostic importance of $\mathrm{Cl}$ and Pra over $\bar{P}$ pa. In

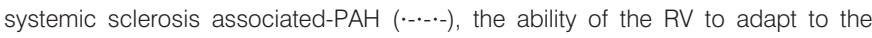
increasing PVR appears limited, therefore, the heart fails at lower PVR [7]. The aim of specific RV-therapies (- - - ) is to improve the ability of the heart to adapt to its afterload. Ref.: reference/normal value.

Current PAH medication (prostacyclines, endothelin receptor blockers, phosphodiesterase (PDE)-5 inhibitors and calcium antagonists) focuses on controlling the excessive vascular remodelling typical for $\mathrm{PAH}$, resulting in a reduction in $\mathrm{RV}$ load [11]. Their cardiac-specific effects on RV adaptation and remodelling have not really been studied yet, but they are most probably of limited clinical relevance as we will demonstrate later. Therefore, there is still unexploited potential for therapies that directly target the right ventricle [12]

In left heart failure (LHF) it is well accepted that the process of cardiac remodelling itself, regardless of the initial cardiac event, is, although compensatory at first, detrimental in the long run [13]. There is now convincing evidence that intervening in the process of remodelling importantly reduces morbidity and mortality in patients with LHF [14, 15]. We hypothesise that the RV remodelling observed in $\mathrm{PAH}$ patients shares important pathophysiological mechanisms with the cardiac remodelling observed in LHF patients. This implies that the adverse RV remodelling could possibly be treated with the same well-established therapies for LHF.

To get better insight into the processes involved, it is essential to clinically distinguish cardiac-specific effects of treatment from their effects on load (pulmonary vasodilation), which also indirectly affect the heart. Therefore, in the first part of this review we will discuss how this separation of effects can be studied, and will evaluate the cardiac-specific effects of current PAH treatments. In the second part of the review we will explore the potential relevance of current evidence-based LHF therapy (table 1) for right heart failure secondary to $\mathrm{PAH}$.

\section{HOW CAN WE DISTINGUISH THE CARDIAC-SPECIFIC EFFECTS OF PAH THERAPY FROM THE PULMONARY VASODILATING EFFECTS?}

The right ventricle and the pulmonary vascular bed are functionally coupled [1, 2]. As a result, it is difficult to distinguish cardiac-specific effects from pulmonary-specific effects of an intervention with the use of standard diagnostic tools (i.e. right heart catheterisation or echocardiography). For example, bosentan treatment has been shown to partially restore cardiac dimensions and function: compared with placebo, bosentan treatment improved cardiac output $\left(0.4 \mathrm{~L} \cdot \mathrm{min}^{-1} \cdot \mathrm{m}^{-2}, \mathrm{p}<0.01\right)$ and $\mathrm{RV} /$ left ventricular $(\mathrm{LV})$ diastolic area ratio $(-0.64, \mathrm{p}<0.01)$ [16]. However, these effects are most probably the result of the decrease in RV load (difference in PVR reduction, bosentan treatment versus placebo: $-415 \pm 99 \mathrm{dyn} \cdot \mathrm{s} \cdot \mathrm{cm}^{-5} ; \mathrm{p}<0.001$ ) [17] and are, therefore, not cardiac specific. Similar observations have been reported for epoprostenol, sildenafil and after successful pulmonary endarterectomy or lung transplantation.

In an experimental setting, this problem can be circumvented by using models with a fixed RV afterload (e.g. pulmonary arterial banding). Herein we describe two methods that are also applicable in a clinical setting.

\section{TABLE 1 Summary of current left (systolic) heart failure therapy}

1. Treat underlying cause when possible, e.g. coronary artery disease and arterial hypertension

2. General measurements, self-care management (avoid drugs that adversely affect the clinical status whenever possible)

3. Diuretics (and moderate salt restriction)

4. $\beta$-blockers (initiated in very low doses, followed by gradual increment)

5. Angiotension-converting enzyme inhibitors (or angiotensin II receptor blockers, if intolerant for angiotension-converting enzyme inhibitors)

6. Aldosterone antagonists (only when renal function is preserved and closely monitored)

7. Exercise training (adjunct to optimal medical therapy)

8. Implantable cardioverter-defibrillator (patients at high-risk for life-threatening arrhythmic disorders)

9. Cardiac resynchronisation therapy (symptomatic heart failure patients despite optimal medical treatment, with signs of cardiac dyssynchrony): digoxin, hydrazaline/nitrate, left ventricle assist devices, heart transplantation 
a)

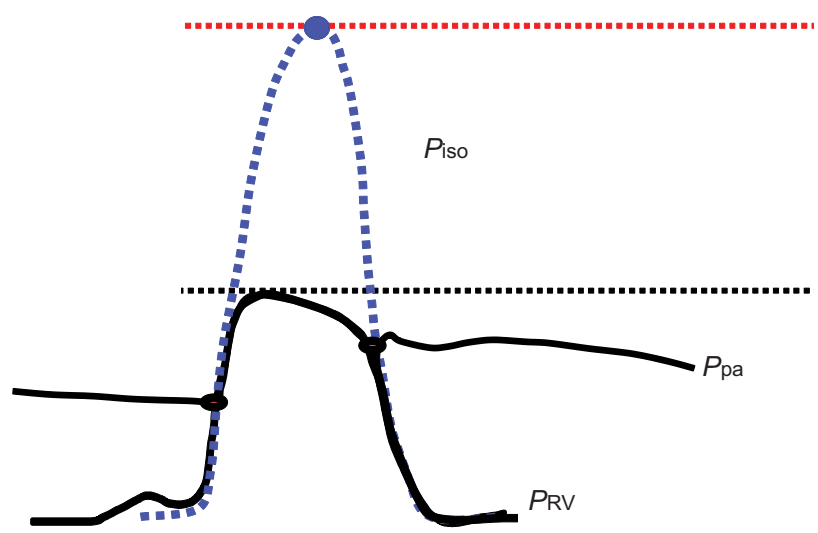

c)

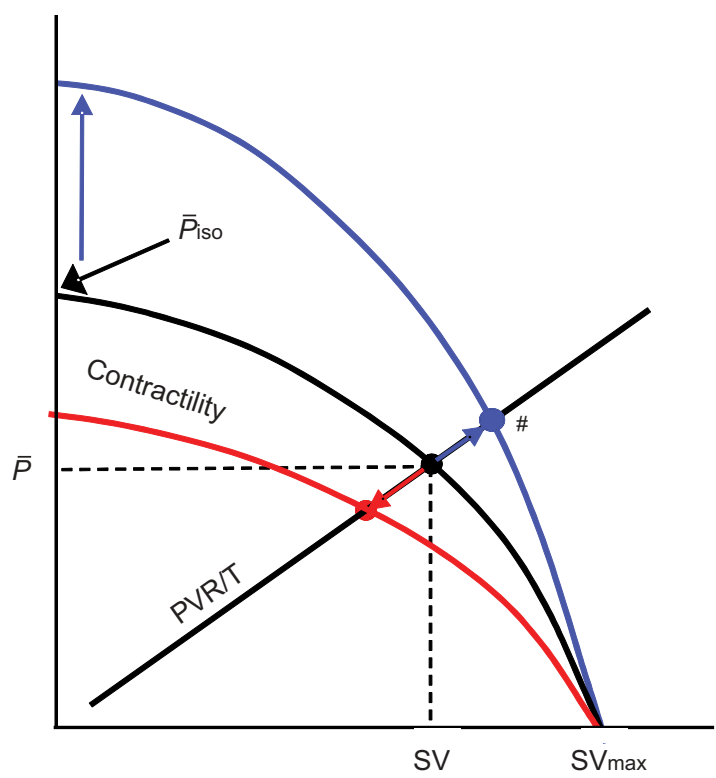

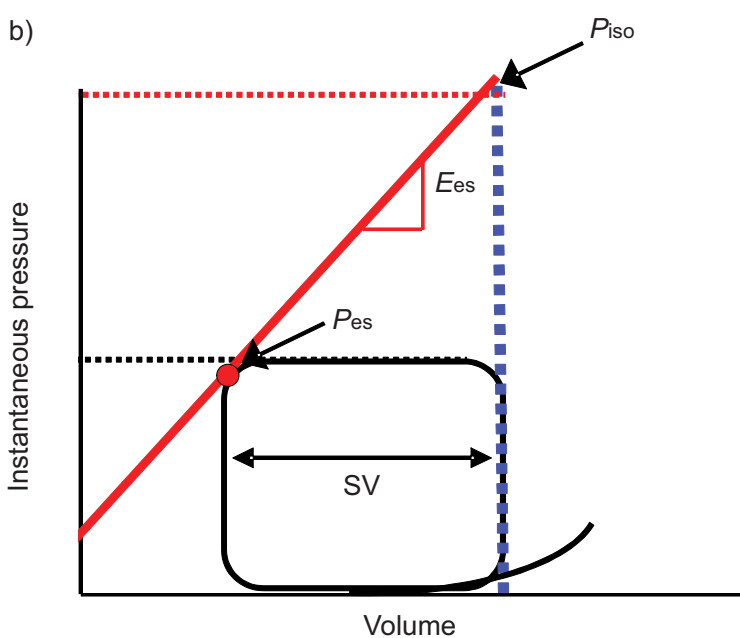

d)

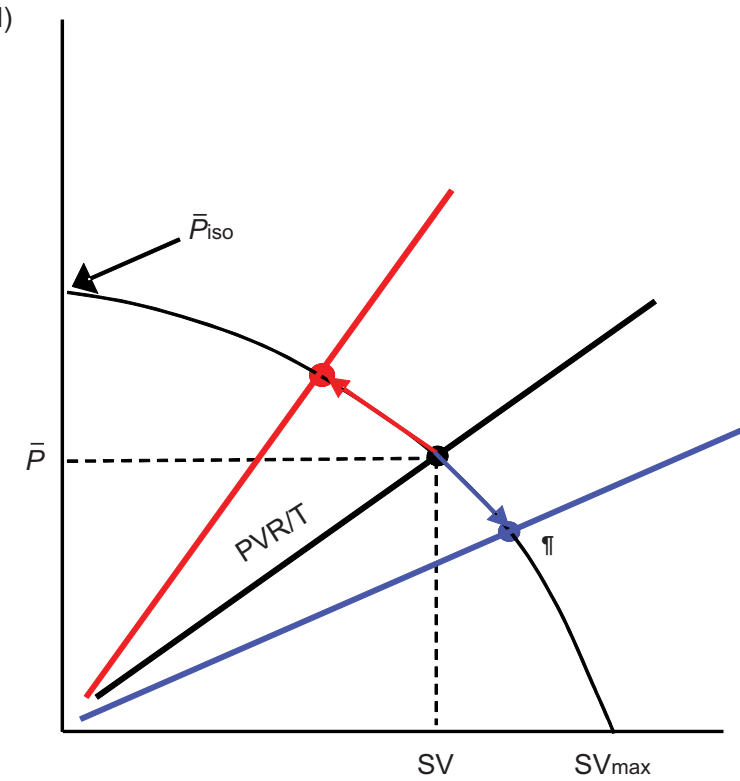

FIGURE 2. Distinguishing cardiac-specific from pulmonary-specific effects in pulmonary hypertension (PAH) patients. a) Pressure curves of the right ventricle (RV) and the main pulmonary artery are shown. Maximal isovolumic pressure is estimated (Piso) by sine wave fit [18]. b) Pressure-volume loops can be constructed from instantaneous pressure and volume measurements by use of conductance catheters. End-systolic elastance (Ees) is considered a load-independent measure of RV contractility and is measured from the slope of the connecting line between end-systolic pressure (Pes) and Piso [19]. c) Increase in contractility. d) Decrease in pulmonary vascular resistance (PVR). An alternative approach for describing heart function is the pump-function graph [20]. Here, average RV pressure versus stroke volume (SV) at steady state are plotted (the working point) and by the same single-beat estimation ( $\bar{P}_{\text {iso) }}$, a pump-function graph is constructed $(-)$. The slope of the line from the origin through the working point is a measure for PVR divided by heart period (PVR/T) and, therefore, a measure for RV afterload. When RV contractility increases (c), this is observed in the pump-function graph by increased $\bar{P}$ iso while SVmax remains unchanged; the new working point has moves to the upper right (\#). When RV afterload is reduced (PVR/T decreases; $d$ ), the pump-function graph remains unchanged, while the new working point moves to the lower right ( $\boldsymbol{\Phi})$. $\bar{P}$ : mean pressure; $P$ pa: pulmonary artery pressure; $P$ RV: RV pressure curve.

\section{Pressure-volume relationship}

It is well accepted that from combined ventricular pressure and volume measurements, parameters of cardiac function and contractility can be derived that are independent of the arterial load. An example of a load-independent parameter of systolic function is the end-systolic elastance (Ees or Emax), which is measured by the slope of the fitted line connecting end-systolic pressure volume points (fig. 2 b). In addition, load-independent parameters of diastolic function can be derived [21, 22]. This method has been used successfully in describing LV performance in multiple disease conditions [23], and more recently its use has been validated in $\mathrm{PAH}$ patients for the right ventricle as well [24]. The construction of pressure-volume loops requires simultaneous measurements of instantaneous pressure- and volume-signals (fig. $2 a$ and b), which can only be obtained using specialised equipment (e.g. conductance catheters). Moreover, to accurately determine Ees, it is necessary to vary cardiac load (usually by a temporary partial occlusion of the inferior vena cava), which might be unacceptable in patients that are haemodynamically compromised, such as PAH patients. Fortunately, mathematical techniques (e.g. single-beat estimation) have been developed that allow 
reasonable estimation of Ees and only require a high-quality RV pressure curve and a reliable stroke volume (SV) measurement during steady state $[18,19]$. Recent studies that compared the separate cardiac and pulmonary effects of norepinephrine, dobutamine and levosimendan in an experimental model for right heart failure are examples of the usefulness of the pressure-volume relationship (including single-beat estimation) $[25,26]$.

\section{Pump-function graph}

An interesting alternative for studying cardiac-specific versus pulmonary-specific effects is the pump-function graph [20, 22] A major advantage of this method is that only instantaneous pressure and average flow measurements suffice, and that its analysis does not require instantaneous volume signals. Average RV pressure is plotted against SV (the working point) and, using the same single-beat estimation as discussed above, a pump-function graph can be constructed (fig. $2 \mathrm{c}$ and d). An increase in mean isovolumic pressure while SVmax remains unchanged (fig. 2) indicates improved cardiac contractility: in this case the new working point moves to the upper right (fig. 2c). A change in cardiac load has a different effect: when load decreases by pulmonary vasodilation (and cardiac contractility remains unchanged) the working point moves to the lower right. With the use of the pump-function graph, we recently demonstrated lower cardiac contractility in systemic sclerosis-associated PAH compared with idiopathic PAH, which could well explain the patients' worse prognosis despite lower PVR [7].

When studying chronic (as opposed to acute) effects of an intervention, both methods (pressure-volume loops and pumpfunction graph) may be insufficient due to RV remodelling. However, they can be further refined by incorporating measures of $R V$ remodelling ( $R V$ wall thickness and $R V$ diameter) in the analysis, in which case RV wall stress ( $\sigma$; estimated by Laplace's law) is used instead of RV pressure [22]. We conclude that by an integral approach, it is certainly possible to distinguish the cardiac-specific from the pulmonary-specific effects of an intervention in PAH patients [22].

Often, it is very difficult to distinguish the cardiac- from the pulmonary-specific effects of PAH therapy in patients. For this purpose, the pressure-volume loop and the pump-function graph have been developed. We propose the use of the pumpfunction graph over the pressure-volume loop as it is more easily obtained in patients using routine RV catheterisation.

\section{CARDIAC EFFECTS OF CURRENT PAH MEDICATION}

The cardiac-specific effects of current PAH therapies, in contrast to their pulmonary-vasodilating effects, have only been investigated in a small number of studies. The few relevant experimental studies are discussed first.

\section{Experimental studies}

ZIERER et al. [27] investigated the effects of diltiazem (a calciumchannel blocker) on RV function in a chronic model of RV pressure overload, using pressure-volume analysis. Administration of diltiazem during constant RV afterload acutely depressed cardiac output, and this was mainly related to depressed right atrial function and RV filling. KERBAUL et al. [28] investigated the effects of prostacyclines in an acute model of RV pressure overload, also using pressure-volume analysis. Epoprostenol improved cardiac output, and this was explained by a marked decrease in RV afterload without detectable changes in RV contractility. These observations have been confirmed by Rex et al. [29]. Two recent papers studied the effects of chronic treatment of sildenafil in a model where RV pressure overload was induced by pulmonary artery banding $[30,31]$. Both studies reported an increase in RV hypertrophy and/or improvement of RV function, which implies that there is a direct effect of sildenafil on the heart. Prior to this, NAGENDRAN et al. [32] reported upregulation of PDE-5 in hypertrophied, but not in normal, rat and human RV myocardium and also demonstrated acute inotropic effects of sildenafil in the isolated Langendorff-perfused heart. In summary, experimental data suggest acute detrimental effects of calcium-channel blockers, a neutral effect of prostacyclines and possibly beneficial cardiac-specific effects of sildenafil on RV function and RV remodelling. Currently, no (experimental) data is available on the cardiac-specific effects of endothelin receptor blockers on the right ventricle in the setting of $\mathrm{PAH}$. To date, these substances have only been evaluated in models in which RV afterload was not fixed.

\section{Meta-analysis of clinical studies}

To the best of our knowledge, no clinical studies exist that specifically separated the cardiac from pulmonary effects of current PAH therapies. Therefore, we re-evaluated all placebocontrolled randomised clinical trials in $\mathrm{PAH}$ that included serial invasive haemodynamic data, recently summarised by GALIE et al. [33], by use of the pump-function graph (fig. 3). $\bar{P}$ pa was used as a surrogate measure for mean RV pressure, SV indexed for body surface area $\left(\mathrm{SV}_{\mathrm{i}}\right)$ was recalculated by dividing cardiac output by heart rate and body surface area (estimated as $1.82 \mathrm{~m}^{2}$ if not reported). Concomitant evaluation of the haemodynamic changes in $\bar{P}$ pa and SVi by the pumpfunction graph, during a typical study period of 12 weeks (range 8 weeks to 12 months), suggests that current $\mathrm{PAH}$ therapies have predominantly pulmonary vasodilating effects. This is highlighted by comparing figure 3 with the situation in figure $2 \mathrm{~d}$. Although future clinical studies specifically designed to address this issue are necessary, this observation demonstrates that there is a strong rationale for developing novel $\mathrm{PAH}$ therapies that specifically target the right ventricle [12].

Right heart function is the main determinant of prognosis in PAH. Current medication (endothelin receptor blockers, PDE-5 inhibitors and prostacyclines) appears to have limited cardiacspecific effects (when analysed by an RV pump-function graph). Novel therapies that specifically improve right heart function in $\mathrm{PAH}$ are needed.

\section{RELEVANCE OF LHF THERAPIES FOR PAH-RELATED RIGHT HEART FAILURE}

The cornerstones of current (systolic) LHF therapy are: (loop)diuretics; a $\beta$-blocker; and angiotensin-converting enzyme (ACE) inhibitors, or angiotensin II receptor blockers if ACE inhibitors are not tolerated (table 1). In case of persisting symptoms, an aldosterone antagonist or an angiotensin blocker is added, if the patient's renal function permits. Exercise training is regarded an adjuvant therapy. For selected LHF patients, an implantable cardioverter-defibrillator and/or 


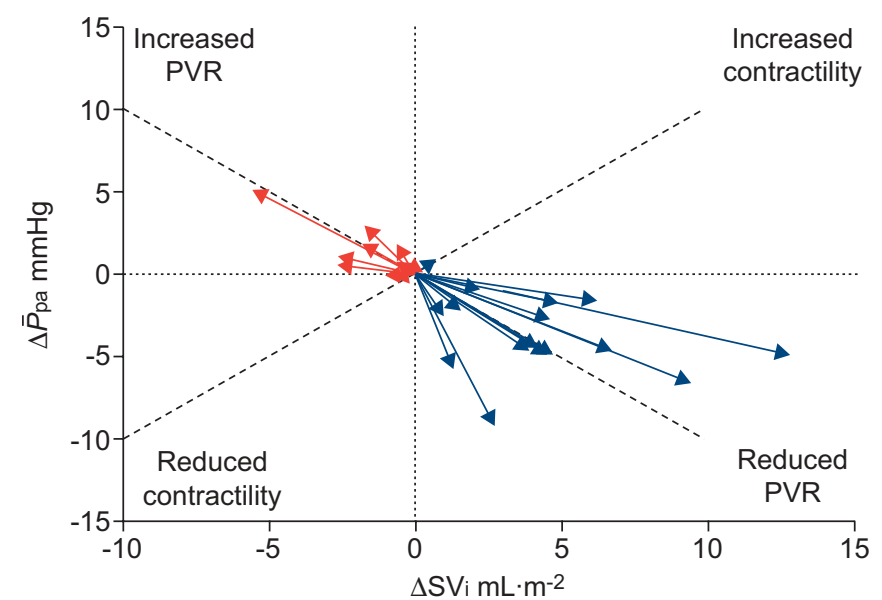

FIGURE 3. Meta-analysis of pulmonary arterial hypertension (PAH) trials by pump function. Each arrow shows the general absolute change in indexed stroke volume $\left(\Delta \mathrm{SV}_{\mathrm{i}}\right)$ and mean pulmonary artery pressure $(\Delta \overline{\mathrm{P}} \mathrm{pa}$; as a surrogate measure for mean right ventricle pressure) per study group of all placebo controlled randomised clinical trials in PAH reporting serial haemodynamic measurements [33]. A decrease in SVi was always accompanied by an increase in $\bar{P}_{p a}$ in the placebo group (red arrows), implying an increase in pulmonary vascular resistance (PVR) without relevant changes in cardiac contractility. For the intervention groups (blue arrows), an increase in SVi was always accompanied by a decrease in $\bar{P}$ pa, implying reduction in PVR without important changes in cardiac contractility. Therefore, current PAH medications predominantly have pulmonary vasodilating effects with only limited cardiac-specific effects.

cardiac resynchronisation therapy can be considered. These therapies are well-established and are based on numerous well-designed randomised controlled trials (more details on current LHF therapy can be found in current guidelines [14, 15]). Of note, clinical benefit in these trials was demonstrated irrespective of the aetiology of LHF. This supports the current idea that the process of cardiac remodelling, after the initial hit, is similar and independent of its cause (e.g. ischaemia or hypertension) [13]. However, it has been argued that therapy efficacy might be different in systolic versus diastolic heart failure (different LHF phenotype) [34]. Therefore, as the cardiac remodelling observed in $\mathrm{PAH}$ patients with right heart failure is comparable to that of systolic LHF (reduced ejection fraction and ventricular dilatation) [10], only these recommendations will be discussed in this article.

It is tempting to extrapolate the LHF recommendations to right heart failure, even though there are important structural, functional and developmental differences between the left and right ventricle [1, 2]. Nevertheless, there is already some overlap in recommendations between the LHF and PAH guidelines [3, 4, 14, 15], which suggests that, at least from a therapeutic perspective, there might be some interesting similarities. For example, loop diuretics are widely used to achieve fast symptomatic relief, both in PAH as well as in LHF. In addition, nowadays, moderate exercise training is accepted as an adjuvant therapy for PAH patients that are clinically stable and under optimal medical treatment [35-37].

Since loop diuretics and exercise training are already part of the recommendations of current PAH guidelines, we will not discuss these therapeutic modalities any further here. We will also not discuss therapies for LHF that are still experimental. Instead, this review will focus on the clinical potential of: 1) $\beta$ blockers as modulators of the sympathetic nervous system; 2) ACE inhibitors, angiotensin blockers and aldosterone antagonists as modulators of the renin-angiotensin-aldosterone system (RAAS); and 3) the potential of electrical cardiac interventions, such as implantable cardioverter-defibrillators and cardiac resynchronisation therapy, as novel add-on therapies for PAH (fig. 4). Because there are hardly any prospective controlled data available that investigated the relevance of these LHF therapies in PAH, we will mainly focus on the relevance of the underlying pathophysiological mechanisms for PAH that are affected by these interventions.

\section{NEUROHUMORAL ACTIVATION AND PAH}

The combined use of a $\beta$-blocker (more specifically bisoprolol, carvedilol or sustained released metoprolol) with either an ACE inhibitor, angiotensin blocker and/or an aldosterone antagonist, in addition to symptomatic treatment by loop diuretics, significantly reduces morbidity and mortality in LHF $[14,15]$. These medications modulate the underlying "neurohumoral activation", which is nowadays considered pathological in the long run, as they promote cardiac remodelling and progression of the disease $[13,38]$. Neurohumoral activation in LHF can be seen as a state in which neural and hormonal systems designed to maintain adequate organ perfusion are increased to excessively high levels. This activation includes many components, of which the sympathetic nervous system and RAAS are, from a therapeutic perspective, the most relevant [14, 15, 38].

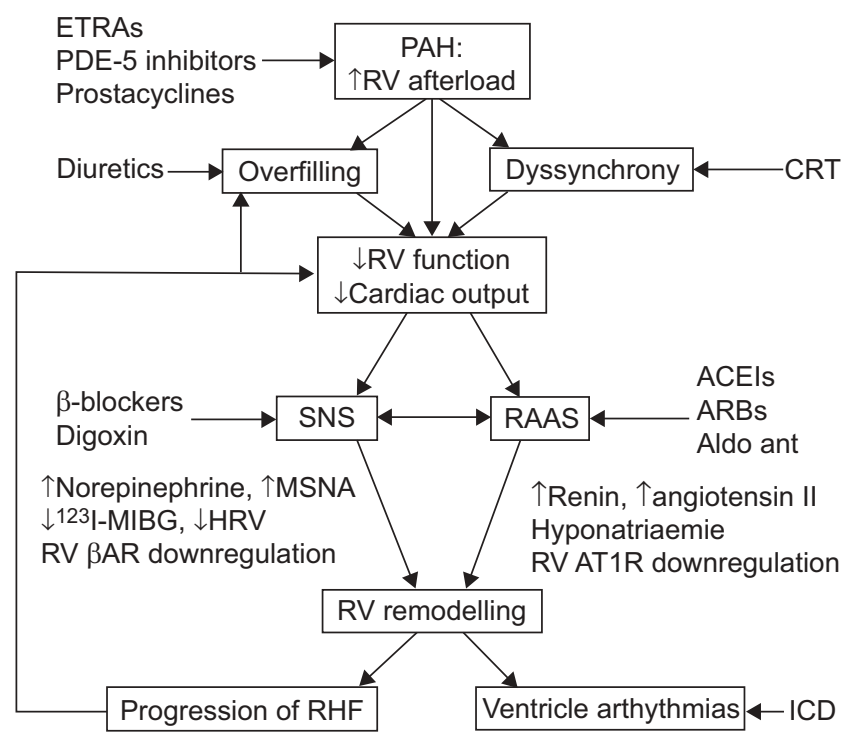

FIGURE 4. Schematic diagram showing as yet unexplored pathophysiological mechanisms in pulmonary arterial hypertension (PAH). RV: right ventricular; ETRAs: endothelin receptor antagonists; PDE-5: phosphodiesterase-5; CRT: cardiac resynchronisation therapy; SNS: sympathetic nervous system; RAAS: reninangiotensin-aldosterone system; ACEls: angiotensin-converting enzyme inhibitors; ARBs: angiotension receptor blockers; MSNA: muscle sympathetic nervous activity; HRV: heart rate variability; $\beta$ AR: cardiomyocyte $\beta_{1}$-adrenergic receptor; AT1R cardiomyocyte angiotensin type 1 receptor; Aldo ant: aldosterone antagonist; RHF: right heart failure; ICD: implantable cardioverter defibrillator. 


\section{Sympathetic nervous system}

Autonomic dysbalance with dominance of the sympathetic system already occurs at early stages of LHF [39], and is mainly attributed to reduced baroreceptor discharge. Baroreceptors, mainly located in the aortic arch, carotid arteries and the left ventricle, are normally triggered by mechanical stretch and respond by tonically inhibiting the central sympathetic neural outflow. In the case of LHF, both systemic arterial pressures and baroreceptor sensitivity are reduced. Sympathetic overdrive leads to chronically elevated levels of norepinephrine, resulting in overstimulation and selective downregulation of the cardiac-specific $\beta_{1}$-adrenergic receptors in the left ventricle. This stimulus not only leads to increased cardiac mechanical stress (by inotropic, chronotropic and vasoconstrictive effects), but also has direct cardiotoxic effects [40]. As a result, LV remodelling progresses with further functional deterioration [38]. $\beta$-blockers are able to stop this vicious circle of heart failure by antagonising the $\beta$ adrenergic receptor [41]. Of interest, the therapeutic effects of digoxin are no longer solely attributed to its weak inotropic effects, but also to its modest neurohumoral effects: digoxin indirectly sensitises the cardiac baroreceptor, and in this way reduces sympathetic outflow of the central nervous system [42].

Although sympathetic activity is difficult to measure in the clinical setting, several methods have been developed to demonstrate sympathetic overdrive in LHF patients [39]. Of these, measurements of regional norepinephrine spill-over or microneurography (which directly measures post-ganglionic muscle sympathetic nerve activity (MSNA)) quantify sympathetic activity best. A sophisticated noninvasive alternative is the use of ${ }^{123}$ I-MIBG tracers (heart-to-mediastinum ratio falls when the sympathetic nervous system is chronically activated). A cruder but easy method is the assessment of heart rate variability (which is reduced when the sympathetic nervous system is over-activated).

\section{Renin-angiotensin-aldosterone system}

Another relevant system in this context, closely interrelated with the sympathetic nervous system, is the RAAS [43]. This system is triggered by impaired renal perfusion due to reduced cardiac output. In this case, the juxtaglomular cells in the kidneys react by secreting renin. Renin increases angiotensin I levels, from which angiotensin II is formed by ACE (abundantly present in the lung endothelium). Angiotensin II mediates multiple processes: it is a potent vasoconstrictor and it has inotropic, natriuretic and antidiuretic properties. In the setting of LHF, all impose cardiac stress and are detrimental in the long run. Like norepinephrine, elevated levels of angiotensin II over stimulate and selectively downregulate its angiotensin II type $1\left(\mathrm{AT}_{1}\right)$-receptor in the left ventricle, directly promoting cardiac remodelling. Furthermore, angiotensin II stimulates the secretion of aldosterone as well as vasopressin (also called antidiuretic hormone). Both have natriuretic and antidiuretic effects and also directly promote cardiac remodelling. ACE inhibitors, angiotensin blockers and aldosterone antagonists interfere in this process by suppressing specific components of RAAS [14, 15].

RAAS activity is relatively easily quantified by directly measuring renin or angiotensin II activity in plasma. A simple but indirect alternative to measure chronically activated RAAS is the assessment of hyponatremia [38].

\section{Over-activation of the sympathetic nervous system in PAH}

Since the primary trigger of neurohumoral activation in LHF (reduction in cardiac output) is also an important clinical feature in $\mathrm{PAH}$, one would expect that the sympathetic nervous system and RAAS are highly activated in PAH as well. Indeed, measurements of the sympathetic and RAAS activation in PAH are comparable to those in LHF [44, 45].

Measurements in PAH patients, as in LHF, revealed elevated levels of norepinephrine in plasma [46, 47], although this was not consistently found in other studies [48]. Furthermore, increased MSNA [48] reduced cardiac uptake of ${ }^{123} \mathrm{I}-\mathrm{MIBG}$ [49], reduced heart rate variability [50], and selective downregulation the $\beta_{1}$-adrenergic receptors in the right (but not left) ventricle have been observed in PAH patients [51], which are all indicators of increased sympathetic activity affecting the right ventricle. Moreover, these findings were correlated with disease severity.

RAAS is also involved in PAH-induced right heart failure. FORFIA et al. [52] recently identified hyponatremia, which indirectly indicates RAAS activation as an important independent prognostic factor in PAH. Parameters that more directly measure RAAS activation (increased renin activity, elevated levels of angiotensin II, aldosterone and/or vasopressin) have not been systematically investigated in PAH patients yet. Nevertheless, increased renin activity and elevated aldosterone levels in plasma have been demonstrated in patients with right heart failure due to hypoxic pulmonary hypertension (cor pulmonale) [53], and in different experimental models of $\mathrm{PAH}-$ induced right heart failure [54]. In addition, selective downregulation of the $\mathrm{AT}_{1}$-receptor in the right ventricle has been observed in PAH patients [55].

Taken together, these studies suggest that the sympathetic nervous system and RAAS are highly activated in PAH. But in contrast to LHF, there are only a few clinical studies that have explored the therapeutic potential of neurohumoral modulation. RICH et al. [56] investigated the effect of i.v. administration of digoxin in $\mathrm{PAH}$ patients and found acute improvement in cardiac output with concomitant reduction in norepinephrine levels, comparable to the digoxin effect in LHF.

Interestingly, no clinical trial exists in $\mathrm{PAH}$-induced right heart failure on the effects of $\beta$-blockers, which have more potent effects on the sympathetic nervous system than digoxin. By common clinical consensus, $\beta$-blocker use is even contraindicated. Often this is substantiated by the study of PROVENCHER et al. [57]. They reported significant functional improvement, 2 months after $\beta$-blocker withdrawal in a small series of patients with porto-pulmonary hypertension. However, all patients (treated for prophylaxis of variceal bleeding) were receiving high-dose propanolol or atenolol. These old $\beta$-blockers are also contraindicated for LHF because of their profound myocardial depressive and vasoconstrictive effects, in comparison to newer $\beta$-blockers [41]. Moreover, it is well-known from LHF that acute functional improvements do not invariably lead to favourable changes long-term, and that overall beneficial effects of $\beta$-blockers can typically be expected after chronic use of $\geqslant 3$ months [41]. 
Another (related) argument against $\beta$-blocker use in PAH is the importance of maintenance of RV systolic function. Acute administration of a $\beta$-blocker is known to exacerbate dyspnoea, most likely due to its negative inotropic effects, leading to instant ventriculo-arterial uncoupling [18]. However, this temporary effect might be better tolerated by careful use of selective $\beta$-blockers ("start low, go slow"), as is successfully demonstrated in LHF-patients [14, 15].

Although this is against present consensus, we would like to argue that, like in LHF, the sympathetic nervous system is activated to pathological levels in $\mathrm{PAH}$, and that this could be normalised by careful $\beta$-blocker use. Further (pre-clinical) research is necessary to investigate whether a low-dose of a newer selective $\beta$-blocker might be a tolerable option to abolish the detrimental effects of sympathetic overdrive in $\mathrm{PAH}$.

\section{Activation of RAAS in PAH}

RAAS has long been recognised to play an important role in pulmonary vascular remodelling and pulmonary vasoconstriction $[58,59]$. Therefore, when captopril (the first ACE inhibitor) became commercially available, this new drug was eagerly tested in $\mathrm{PAH}$ patients, for whom no effective treatment existed at that time. In the 1980s, four small case-series (26 patients in total) reported on the haemodynamic effects of captopril in PAH. Three of the studies were positive and found a significant increase in cardiac output $[60,61]$ and exercise capacity [62]. One study, however, did not observe any haemodynamic changes, neither positive nor negative [63]. Surprisingly, no additional clinical studies have been published since. Currently, there is renewed interest in RAAS since the discovery of ACE2, an isoform of ACE with counteractive (protective) actions $[64,65]$. The theoretical beneficial effects of ACE inhibitors, angiotensin blockers and/or aldosteron antagonists on the heart in PAH have not been addressed in patients yet.

Pre-clinical studies using different models of $\mathrm{PAH}$ and right heart failure, however confirmed that the use of ACE inhibitors or angiotensin blockers significantly reduces RV remodelling and improves cardiac function and/or survival [66-69]. We conclude that in PAH, pharmacological interference in RAAS could (partially) reverse pulmonary and cardiac remodelling, which warrants a prospective controlled clinical study of the effects of ACE inhibitors, angiotensin blockers and/or aldosterone antagonists.

There is convincing evidence that, as in LHF, the sympathetic nervous system and RAAS in PAH are highly activated. Wellestablished pharmacological interventions for LHF could, therefore, be relevant for $\mathrm{PAH}$ as well. However, ACE inhibitors, angiotensin II blockers and selective $\beta$-blockade have not been explored in $\mathrm{PAH}$, and clinical investigations of the potential of these drugs are urgently needed. Nevertheless, routine use of these neurohumoral modulators and, in particular, the use of $\beta$-blockers are currently not recommended in $\mathrm{PAH}$, unless future studies can prove that their use in PAH is safe and beneficial.

\section{ELECTRICAL REMODELLING AND PAH}

Implantable cardioverter-defibrillators and cardiac resynchronisation therapy are relatively new therapeutic modalities in LHF management. Major heart failure guidelines contain recommendations on implantable cardioverter-defibrillator use since 2001, but recommendations on resynchronisation therapy have only been incorporated since 2005. For selected LHF patient groups, it is now well-accepted that resynchronisation therapy significantly reduce morbidity, and both cardioverter-defibrillators and resynchronisation therapy significantly reduce mortality, in addition to the beneficial effect of optimal pharmacological LHF treatment $[14,15]$.

\section{Implantable cardioverter-defibrillator}

A cardioverter-defibrillator detects and prematurely terminates malignant and life-threatening ventricular arrhythmias, preventing sudden cardiac death. The incidence of ventricular arrhythmias increases with the progression of LHF. Therefore, implantable cardioverter-defibrillator use is currently recommended as secondary prevention for sudden cardiac death in LHF patients with a (presumed) history of ventricular arrhythmia, or as primary prevention in LHF patients with a severely reduced left ventricular-ejection fraction. In both cases LHF patients must have a reasonable expectation of survival with acceptable functional status of $>1 \mathrm{yr}[14,15]$. The clinical trials, on which these recommendations are based, reported a relative reduction of all-cause mortality after 24 months of $\sim 30 \%$ and an absolute risk reduction of $\sim 5 \%[70,71]$, which implies a number-needed-to-treat of $\sim 20$ patients.

Also in PAH, sudden cardiac death, presumably due to malignant ventricular arrhythmias, has been recognised as an important clinical risk for these patients [72]. As in LHF, markers for an "electrically instable heart", such as prolonged QTc-intervals and increased QT dispersion derived by ECG [73], neurohumoral disturbances (as discussed previously) and increase in cardiac fibrosis [74], have been demonstrated in $\mathrm{PAH}$ patients. However, in contrast to LHF, the actual incidence of events related to ventricular arrhythmias in $\mathrm{PAH}$ is considered to be low. However, the reported percentages of deaths in $\mathrm{PAH}$ attributed to ventricular arrhythmias vary widely, from 8 to $26 \%[8,75]$, and the actual numbers might importantly differ for the different PAH subgroups (e.g. higher for $\mathrm{PAH}$ associated with congenital heart disease, which might be related to the presence of surgical cardiac scars [76]). Moreover, these numbers are based on retrospective studies and were partially obtained before the introduction of PAH specific medications. Systematic prospective clinical studies are necessary to accurately determine the current incidence of sudden cardiac deaths in different subgroups of PAH. These data will allow a crude estimation of the clinical potential of cardioverter-defibrillators in $\mathrm{PAH}$, by calculation of the number-needed-to-treat (extrapolating the effect of cardioverter-defibrillators in LHF). Until then, implantable cardioverter-defibrillators (or pharmacological anti-arrhythmic agents) are generally not recommended as a (primary) preventive measure for sudden cardiac death in PAH patients $[4,72]$.

\section{Supraventricular tachyarrhythmias}

In contrast to ventricular arrhythmias, the incidence of supraventricular arrhythmias seems to be much higher, and they are considered to be an important cause of clinical deterioration in PAH patients. In a retrospective analysis [77], 
an annual incidence of supraventricular tachyarrhythmias in $\mathrm{PAH}$ of $\sim 3 \%$ was found: atrial fibrillation and atrial flutter were equally common. In this study, persistent atrial fibrillation was associated with a very poor prognosis (nine out of 11 PAH patients died within 24 months), which may be explained by worsening of RV function due to the loss of atrial "kick" to ventricular filling. Maintenance of sinus rhythm is, therefore, currently considered an important treatment goal in PAH [4]. However, this is in contrast to the clinical experience in LHF. The treatment strategy of rhythm-control, compared with ratecontrol (which is acceptance of atrium fibrillation and lowering of the ventricular response-rate in combination with adequate anticoagulation), had no superior effect on survival, whereas it required more hospitalisation because of the need for repeated cardioversion [78, 79]. We fully support current recommendations to restore and maintain sinus rhythm in $\mathrm{PAH}$ patients if possible, by lack of prospective and controlled data. Future trials, however, are necessary to test the validity of this treatment strategy.

\section{Cardiac resynchronisation therapy}

Cardiac dyssynchrony in LHF is characterised by regional differences in electrical and/or mechanical activation of the left ventricle (usually a delay in activation of the LV free wall in relation to the interventricular septum). Dyssynchrony results in inefficient pumping of the left ventricle, and further clinical deterioration. Cardiac resynchronisation therapy can acutely restore synchrony of LV contraction, thereby improving overall LV (systolic) performance. In the long run, resynchronisation therapy leads to reversed cardiac remodelling, resulting in an even further improvement of LV performance. Although the current clinical selection criteria for resynchronisation therapy (wide QRS complex on ECG) sub-optimally predict clinical benefit for the individual LHF patient, resynchronisation therapy has been shown to significantly reduce morbidity and mortality, and is now a well-established treatment modality in LHF $[14,15]$.

Ventricular dyssynchrony is often also observed in progressive stages of PAH-induced right heart failure [80, 81]. Mechanical interventricular dyssynchrony in $\mathrm{PAH}$ (which is clinically easily recognised by the paradoxical bulging of the interventricular septum) is associated with impaired RV systolic function. Furthermore, through septum bulging, ventricular dyssynchrony is thought to impair $\mathrm{LV}$ diastolic function as well [82, 83]. Resynchronisation of the right ventricle could, therefore, be of clinical benefit in PAH. However, we recently demonstrated that LV and RV dyssynchrony are essentially different: the origin of PAH-related ventricular dyssynchrony lies in regional differences in the duration of the contraction, rather than regional differences in onset of the contraction (e.g. due to a conductance delay); and is highly afterload dependent [83, 84].

Previously, successful application of cardiac resynchronisation therapy has been demonstrated in patients with $\mathrm{PAH}$ associated with congenital heart disease [85]. However, these patients display a "LHF-like" dyssynchrony due to a complete right bundle branch block as a (late) complication of cardiac surgery and are, therefore, not representative for the PAH population in general. We recently explored the clinical potential of resynchronisation therapy in an experimental model of PAH-induced right heart failure, in the absence of conduction disturbances [84]. We found that pre-excitation of the RV free wall resulted in improved RV systolic function and reduced adverse LV diastolic interaction. Interestingly, these findings have recently been confirmed by HARDZIYENKA et al. [86] in a study with patients suffering from right heart failure and ventricular dyssynchrony secondary to chronic thromboembolic pulmonary hypertension. A cohort of 67 patients was pre-operatively screened by standard tissue-Doppler echocardiography and seven patients were selected for a temporary pacing protocol, based on the presence of large diastolic interventricular delay (as a quantification of PAH-related ventricular dyssynchrony). Resynchronisation therapy acutely reduced ventricular dyssynchrony, enhanced RV contractility and LV diastolic filling, and resulted in an improvement of SV of $>10 \%$. These promising results warrant further investigations of cardiac resynchronisation therapy as a novel treatment for right heart failure secondary to $\mathrm{PAH}$ [12], that should focus on its long-term effects, and on the identification of robust selection criteria for $\mathrm{PAH}$ patients that could profit most from cardiac resynchronisation therapy.

In $\mathrm{PAH}$, the incidence of malignant ventricular arrhythmias is considered low, but this observation needs prospective validation. For now, the use of implantable cardioverterdefibrillators is not recommended for $\mathrm{PAH}$ patients. Supraventricular tachyarrhythmias often lead to clinical deterioration. Based on retrospective data, maintenance of sinus rhythm is an important treatment goal, but this preference of rhythm- over rate-control needs be confirmed in prospective controlled studies, especially because this is opposite to the experiences in left heart failure. Cardiac resynchronisation therapy emerges as a promising new treatment modality. Prospective controlled trials are necessary to study its long-term effects and to identify robust selection criteria.

\section{CONCLUSIONS AND FUTURE DIRECTIONS}

In this review we investigated the potential applicability of current LHF therapy for the treatment of PAH-induced right heart failure. Based on available literature, we conclude that: LHF and right heart failure share important underlying pathophysiological mechanisms that are amenable for treatment (fig. 4); however, clinical experience with current LHF treatments in the setting of PAH is very limited.

This discrepancy is intriguing, and we can only speculate about its reasons. First, it is difficult to separate cardiac- from pulmonary-specific effects of therapeutic interventions in $\mathrm{PAH}$ patients. As a solution, we propose the use of the pumpfunction graph. Secondly, PAH remains a rare disease where many other clinical trials have been undertaken in the last two 20 yrs [33]. Thirdly, until recently right heart failure was regarded as an inevitable final consequence of $\mathrm{PAH}$, whereas it $\mathrm{S}$ now the right ventricle which is considered a potential therapeutic target [12].

So, how do we move forward? Solid clinical evidence is essential before LHF therapy can be implemented in $\mathrm{PAH}$ management. Therefore, phase I/II trials need to be conducted first, which must provide insights in safety, tolerability and efficacy of LHF therapy in PAH. Subsequently, randomised clinical trials should be performed that compare current $\mathrm{PAH}$ 
therapy with and without add-on LHF therapy. An important aspect is sufficient duration of the trial: the experiences in LHF would predict that reversal of cardiac remodelling requires more than the typical 12-week trial duration. In addition, the question remains as to which end-point to choose in these types of studies: classical end-points in PAH trials, such as 6min walking distance, might not be sensitive enough, and direct measures for RV remodelling and function are possibly more appropriate. However, the most ideal end-point, mortality, might be too stringent and will require inclusion of unrealistically high numbers of patients [87].

To conclude, well-designed clinical studies are warranted, as they might provide supporting evidence for the use of novel therapeutic modalities that are relatively easily available to treat this devastating disease. Future investigations will reveal whether going "left" is a step in the "right" direction.

\section{SUPPORT STATEMENT}

M.L. Handoko (Mozaïek 017.002.122) and A. Vonk-Noordegraaf (Vidi 917.96.306) were supported by the Netherlands Organisation for Scientific Research (The Hague, The Netherlands).

\section{STATEMENT OF INTEREST}

C.P. Allaart received a consulting fee from Biotronik. C.P. Allaart has attended several cardiology conferences that were partly funded by several major pacemaker/ICD companies.

\section{REFERENCES}

1 Haddad F, Hunt SA, Rosenthal DN, et al. Right ventricular function in cardiovascular disease, part I: Anatomy, physiology, aging, and functional assessment of the right ventricle. Circulation 2008; 117: 1436-1448.

2 Haddad F, Doyle R, Murphy DJ, et al. Right ventricular function in cardiovascular disease, part II: pathophysiology, clinical importance, and management of right ventricular failure. Circulation 2008; 117: 1717-1731.

3 McLaughlin VV, Archer SL, Badesch DB, et al. ACCF/AHA 2009 expert consensus document on pulmonary hypertension a report of the American College of Cardiology Foundation Task Force on Expert Consensus Documents and the American Heart Association developed in collaboration with the American College of Chest Physicians; American Thoracic Society, Inc.; and the Pulmonary Hypertension Association. J Am Coll Cardiol 2009; 53: 1573-1619.

4 Galie N, Hoeper MM, Humbert M, et al. Guidelines for the diagnosis and treatment of pulmonary hypertension. The Task Force for the Diagnosis and Treatment of Pulmonary Hypertension of the European Society of Cardiology (ESC) and the European Respiratory Society (ERS), endorsed by the International Society of Heart and Lung Transplantation (ISHLT). Eur Respir J 2009; 30: 2493-2537.

5 Lankhaar JW, Westerhof N, Faes TJ, et al. Quantification of right ventricular afterload in patients with and without pulmonary hypertension. Am J Physiol Heart Circ Physiol 2006; 291: H1731-H1737.

6 McLaughlin VV, McGoon MD. Pulmonary arterial hypertension. Circulation 2006; 114: 1417-1431.

7 Overbeek MJ, Lankhaar JW, Westerhof N, et al. Right ventricular contractility in systemic sclerosis-associated and idiopathic pulmonary arterial hypertension. Eur Respir J 2008; 31: 1160-1166.

8 D'Alonzo GE, Barst RJ, Ayres SM, et al. Survival in patients with primary pulmonary hypertension. Results from a national prospective registry. Ann Intern Med 1991; 115: 343-349.
9 Sandoval J, Bauerle O, Palomar A, et al. Survival in primary pulmonary hypertension. Validation of a prognostic equation. Circulation 1994; 89: 1733-1744.

10 van Wolferen SA, Marcus JT, Boonstra A, et al. Prognostic value of right ventricular mass, volume, and function in idiopathic pulmonary arterial hypertension. Eur Heart J 2007; 28: 1250-1257.

11 Humbert M, Sitbon O, Simonneau G. Treatment of pulmonary arterial hypertension. N Engl J Med 2004; 351: 1425-1436.

12 Ghofrani HA, Barst RJ, Benza RL, et al. Future perspectives for the treatment of pulmonary arterial hypertension. J Am Coll Cardiol 2009; 54: Suppl. 1, S108-S117.

13 Cohn JN, Ferrari R, Sharpe N. Cardiac remodelling - concepts and clinical implications: a consensus paper from an international forum on cardiac remodelling. Behalf of an International Forum on Cardiac remodelling. J Am Coll Cardiol 2000; 35: 569-582.

14 Hunt SA, Abraham WT, Chin MH, et al. 2009 focused update incorporated into the ACC/AHA 2005 Guidelines for the Diagnosis and Management of Heart Failure in Adults: a report of the American College of Cardiology Foundation/American Heart Association Task Force on Practice Guidelines: developed in collaboration with the International Society for Heart and Lung Transplantation. Circulation 2009; 119: e391-e479.

15 Dickstein K, Cohen-Solal A, Filippatos G, et al. ESC Guidelines for the diagnosis and treatment of acute and chronic heart failure 2008: the Task Force for the Diagnosis and Treatment of Acute and Chronic Heart Failure 2008 of the European Society of Cardiology. Developed in collaboration with the Heart Failure Association of the ESC (HFA) and endorsed by the European Society of Intensive Care Medicine (ESICM). Eur Heart J 2008; 29: 2388-2442.

16 Galie N, Hinderliter AL, Torbicki A, et al. Effects of the oral endothelin-receptor antagonist bosentan on echocardiographic and doppler measures in patients with pulmonary arterial hypertension. J Am Coll Cardiol 2003; 41: 1380-1386.

17 Channick RN, Simonneau G, Sitbon O, et al. Effects of the dual endothelin-receptor antagonist bosentan in patients with pulmonary hypertension: a randomised placebo-controlled study. Lancet 2001; 358: 1119-1123.

18 Brimioulle S, Wauthy P, Ewalenko P, et al. Single-beat estimation of right ventricular end-systolic pressure-volume relationship. Am J Physiol Heart Circ Physiol 2003; 284: H1625-H1630.

19 Sunagawa K, Yamada A, Senda Y, et al. Estimation of the hydromotive source pressure from ejecting beats of the left ventricle. IEEE Trans Biomed Eng 1980; 27: 299-305.

20 Elzinga G, Westerhof N. How to quantify pump function of the heart. The value of variables derived from measurements on isolated muscle. Circ Res 1979; 44: 303-308.

21 Suga H, Sagawa K, Shoukas AA. Load independence of the instantaneous pressure-volume ratio of the canine left ventricle and effects of epinephrine and heart rate on the ratio. Circ Res 1973; 32: 314-322.

22 Westerhof N, Stergiopulos N, Noble MIM, eds, Snapshots of Hemodynamics: An Aid for Clinical Research and Graduate Education. New York, Springer, 2005.

23 Burkhoff D, Mirsky I, Suga H. Assessment of systolic and diastolic ventricular properties via pressure-volume analysis: a guide for clinical, translational, and basic researchers. Am J Physiol Heart Circ Physiol 2005; 289: H501-H512.

24 Kuehne T, Yilmaz S, Steendijk P, et al. Magnetic resonance imaging analysis of right ventricular pressure-volume loops: in vivo validation and clinical application in patients with pulmonary hypertension. Circulation 2004; 110: 2010-2016.

25 Kerbaul F, Rondelet B, Demester JP, et al. Effects of levosimendan versus dobutamine on pressure load-induced right ventricular failure. Crit Care Med 2006; 34: 2814-2819.

26 Kerbaul F, Rondelet B, Motte S, et al. Effects of norepinephrine and dobutamine on pressure load-induced right ventricular failure. Crit Care Med 2004; 32: 1035-1040. 
27 Zierer A, Voeller RK, Melby SJ, et al. Impact of calcium-channel blockers on right heart function in a controlled model of chronic pulmonary hypertension. Eur J Anaesthesiol 2009; 26: 253-259.

28 Kerbaul F, Brimioulle S, Rondelet B, et al. How prostacyclin improves cardiac output in right heart failure in conjunction with pulmonary hypertension. Am J Respir Crit Care Med 2007; 175: 846-850.

29 Rex S, Missant C, Segers $\mathrm{P}$, et al. Epoprostenol treatment of acute pulmonary hypertension is associated with a paradoxical decrease in right ventricular contractility. Intensive Care Med 2008; 34: 179-189.

30 Schafer S, Ellinghaus P, Janssen W, et al. Chronic inhibition of phosphodiesterase 5 does not prevent pressure-overload-induced right-ventricular remodelling. Cardiovasc Res 2009; 82: 30-39.

31 Andersen A, Nielsen JM, Peters CD, et al. Effects of phosphodiesterase- 5 inhibition by sildenafil in the pressure overloaded right heart. Eur J Heart Fail 2008; 10: 1158-1165.

32 Nagendran J, Archer SL, Soliman D, et al. Phosphodiesterase type 5 is highly expressed in the hypertrophied human right ventricle, and acute inhibition of phosphodiesterase type 5 improves contractility. Circulation 2007; 116: 238-248.

33 Galie N, Manes A, Negro L, et al. A meta-analysis of randomized controlled trials in pulmonary arterial hypertension. Eur Heart J 2009; 30: 394-403.

34 Paulus WJ, van Ballegoij JJM. Treatment of heart failure with normal ejection fraction: an inconvenient truth! J Am Coll Cardiol 2010; [Epub ahead of print DOI: 10.1016/j.jacc.2009.06.067].

35 Mereles D, Ehlken N, Kreuscher S, et al. Exercise and respiratory training improve exercise capacity and quality of life in patients with severe chronic pulmonary hypertension. Circulation 2006; 114: 1482-1489.

36 de Man FS, Handoko ML, Groepenhoff H, et al. Effects of exercise training in patients with idiopathic pulmonary arterial hypertension. Eur Respir J 2009; 34: 669-675.

37 Handoko ML, de Man FS, Happe CM, et al. Opposite effects of training in rats with stable and progressive pulmonary hypertension. Circulation 2009; 120: 42-49.

38 Packer M. The neurohormonal hypothesis: a theory to explain the mechanism of disease progression in heart failure. J Am Coll Cardiol 1992; 20: 248-254.

39 Triposkiadis F, Karayannis G, Giamouzis G, et al. The sympathetic nervous system in heart failure physiology, pathophysiology, and clinical implications. J Am Coll Cardiol 2009; 54: 1747-1762.

40 Engelhardt S, Hein L, Wiesmann F, et al. Progressive hypertrophy and heart failure in $\beta 1$-adrenergic receptor transgenic mice. Proc Natl Acad Sci USA 1999; 96: 7059-7064.

41 Bristow MR. $\beta$-adrenergic receptor blockade in chronic heart failure. Circulation 2000; 101: 558-569.

42 Gheorghiade M, Ferguson D. Digoxin. A neurohormonal modulator in heart failure? Circulation 1991; 84: 2181-2186.

43 Baker KM, Booz GW, Dostal DE. Cardiac actions of angiotensin II: role of an intracardiac renin-angiotensin system. Annu Rev Physiol 1992; 54: 227-241.

44 Kurzyna M, Torbicki A. Neurohormonal modulation in right ventricular failure. Eur Heart J Suppl 2007; 9: Suppl. H, H35-H40.

45 Schrier RW, Bansal S. Pulmonary hypertension, right ventricular failure, and kidney: different from left ventricular failure? Clin J Am Soc Nephrol 2008; 3: 1232-1237.

46 Nootens M, Kaufmann E, Rector $\mathrm{T}$, et al. Neurohormonal activation in patients with right ventricular failure from pulmonary hypertension: relation to haemodynamic variables and endothelin levels. J Am Coll Cardiol 1995; 26: 1581-1585.

47 Nagaya N, Nishikimi T, Uematsu M, et al. Plasma brain natriuretic peptide as a prognostic indicator in patients with primary pulmonary hypertension. Circulation 2000; 102: 865-870.

48 Velez-Roa S, Ciarka A, Najem B, et al. Increased sympathetic nerve activity in pulmonary artery hypertension. Circulation 2004; 110: $1308-1312$.
49 Morimitsu T, Miyahara Y, Sinboku H, et al. Iodine-123-metaiodobenzylguanidine myocardial imaging in patients with right ventricular pressure overload. J Nucl Med 1996; 37: 1343-1346.

50 Wensel R, Jilek C, Dorr M, et al. Impaired cardiac autonomic control relates to disease severity in pulmonary hypertension. Eur Respir J 2009; 34: 895-901.

51 Bristow MR, Minobe W, Rasmussen R, et al. $\beta$-adrenergic neuroeffector abnormalities in the failing human heart are produced by local rather than systemic mechanisms. J Clin Invest 1992; 89: 803-815.

52 Forfia PR, Mathai SC, Fisher MR, et al. Hyponatremia predicts right heart failure and poor survival in pulmonary arterial hypertension. Am J Respir Crit Care Med 2008; 177: 1364-1369.

53 Anand IS, Chandrashekhar Y, Ferrari R, et al. Pathogenesis of congestive state in chronic obstructive pulmonary disease. Studies of body water and sodium, renal function, haemodynamics, and plasma hormones during edema and after recovery. Circulation 1992; 86: 12-21.

54 Watkins L Jr, Burton JA, Haber E, et al. The renin-angiotensinaldosterone system in congestive failure in conscious dogs. J Clin Invest 1976; 57: 1606-1617.

55 Asano K, Dutcher DL, Port JD, et al. Selective downregulation of the angiotensin II AT1-receptor subtype in failing human ventricular myocardium. Circulation 1997; 95: 1193-1200.

56 Rich S, Seidlitz M, Dodin E, et al. The short-term effects of digoxin in patients with right ventricular dysfunction from pulmonary hypertension. Chest 1998; 114: 787-792.

57 Provencher S, Herve P, Jais X, et al. Deleterious effects of $\beta$ blockers on exercise capacity and haemodynamics in patients with portopulmonary hypertension. Gastroenterology 2006; 130: 120-126.

58 Cargill RI, Lipworth BJ. The role of the renin-angiotensin and natriuretic peptide systems in the pulmonary vasculature. $\mathrm{Br} J \mathrm{Clin}$ Pharmacol 1995; 40: 11-18.

59 Qing F, McCarthy TJ, Markham J, et al. Pulmonary angiotensinconverting enzyme (ACE) binding and inhibition in humans. A positron emission tomography study. Am J Respir Crit Care Med 2000; 161: 2019-2025.

60 Niarchos AP, Whitman HH, Goldstein JE, et al. haemodynamic effects of captopril in pulmonary hypertension of collagen vascular disease. Am Heart J 1982; 104: 834-838.

61 Alpert MA, Pressly TA, Mukerji V, et al. Short- and long-term haemodynamic effects of captopril in patients with pulmonary hypertension and selected connective tissue disease. Chest 1992; 102: $1407-1412$.

62 Ikram H, Maslowski AH, Nicholls MG, et al. Haemodynamic and hormonal effects of captopril in primary pulmonary hypertension. Br Heart J 1982; 48: 541-545.

63 Leier CV, Bambach D, Nelson S, et al. Captopril in primary pulmonary hypertension. Circulation 1983; 67: 155-161.

64 Kuba K, Imai Y, Penninger JM. Angiotensin-converting enzyme 2 in lung diseases. Curr Opin Pharmacol 2006; 6: 271-276.

65 Ferreira AJ, Shenoy V, Yamazato Y, et al. Evidence for angiotensinconverting enzyme 2 as a therapeutic target for the prevention of pulmonary hypertension. Am J Respir Crit Care Med 2009; 179: 1048-1054.

66 Okada M, Kikuzuki R, Harada T, et al. Captopril attenuates matrix metalloproteinase-2 and -9 in monocrotaline-induced right ventricular hypertrophy in rats. J Pharmacol Sci 2008; 108: 487-494.

67 Okada M, Harada T, Kikuzuki R, et al. Effects of telmisartan on right ventricular remodelling induced by monocrotaline in rats. J Pharmacol Sci 2009; 111: 193-200.

68 Rouleau JL, Kapuku G, Pelletier S, et al. Cardioprotective effects of ramipril and losartan in right ventricular pressure overload in the rabbit: importance of kinins and influence on angiotensin II type 1 receptor signaling pathway. Circulation 2001; 104: 939-944.

69 Morrell NW, Atochina EN, Morris KG, et al. Angiotensin converting enzyme expression is increased in small pulmonary 
arteries of rats with hypoxia-induced pulmonary hypertension. J Clin Invest 1995; 96: 1823-1833.

70 Moss AJ, Zareba W, Hall WJ, et al. Prophylactic implantation of a defibrillator in patients with myocardial infarction and reduced ejection fraction. N Engl J Med 2002; 346: 877-883.

71 Bardy GH, Lee KL, Mark DB, et al. Amiodarone or an implantable cardioverter-defibrillator for congestive heart failure. $N$ Engl J Med 2005;20, 352: 225-237.

72 Zipes DP, Camm AJ, Borggrefe M, et al. ACC/AHA/ESC 2006 Guidelines for Management of Patients With Ventricular Arrhythmias and the Prevention of Sudden Cardiac Death: a report of the American College of Cardiology/American Heart Association Task Force and the European Society of Cardiology Committee for Practice Guidelines (writing committee to develop Guidelines for Management of Patients With Ventricular Arrhythmias and the Prevention of Sudden Cardiac Death): developed in collaboration with the European Heart Rhythm Association and the Heart Rhythm Society. Circulation 2006; 114: e385-e484.

73 Hong-liang Z, Qin L, Zhi-hong L, et al. Heart rate-corrected QT interval and QT dispersion in patients with pulmonary hypertension. Wien Klin Wochenschr 2009; 121: 330-333.

74 Lowes BD, Minobe W, Abraham WT, et al. Changes in gene expression in the intact human heart. Downregulation of alphamyosin heavy chain in hypertrophied, failing ventricular myocardium. J Clin Invest 1997; 100: 2315-2324.

75 Hoeper MM, Galie N, Murali S, et al. Outcome after cardiopulmonary resuscitation in patients with pulmonary arterial hypertension. Am J Respir Crit Care Med 2002; 165: 341-344.

76 Walsh EP, Cecchin F. Arrhythmias in adult patients with congenital heart disease. Circulation 2007; 115: 534-545.

77 Tongers J, Schwerdtfeger B, Klein G, et al. Incidence and clinical relevance of supraventricular tachyarrhythmias in pulmonary hypertension. Am Heart J 2007; 153: 127-132.

78 Roy D, Talajic M, Nattel S, et al. Rhythm control versus rate control for atrial fibrillation and heart failure. N Engl J Med 2008;19, 358: 2667-2677.

79 Fuster V, Ryden LE, Cannom DS, et al. ACC/AHA/ESC 2006 Guidelines for the Management of Patients with Atrial Fibrillation: a report of the American College of Cardiology/American Heart Association Task Force on Practice Guidelines and the European Society of Cardiology Committee for Practice Guidelines (Writing Committee to Revise the 2001 Guidelines for the Management of Patients With Atrial Fibrillation): developed in collaboration with the European Heart Rhythm Association and the Heart Rhythm Society. Circulation 2006; 114: e257-e354.

80 Roeleveld RJ, Marcus JT, Faes TJ, et al. Interventricular septal configuration at MR imaging and pulmonary arterial pressure in pulmonary hypertension. Radiology 2005; 234: 710-717.

81 Tanaka H, Tei C, Nakao S, et al. Diastolic bulging of the interventricular septum toward the left ventricle. An echocardiographic manifestation of negative interventricular pressure gradient between left and right ventricles during diastole. Circulation 1980; 62: 558-563.

82 Dohi K, Onishi K, Gorcsan J III, et al. Role of radial strain and displacement imaging to quantify wall motion dyssynchrony in patients with left ventricular mechanical dyssynchrony and chronic right ventricular pressure overload. Am J Cardiol 2008; 101: 1206-1212.

83 Marcus JT, Gan CT, Zwanenburg JJ, et al. Interventricular mechanical asynchrony in pulmonary arterial hypertension: leftto-right delay in peak shortening is related to right ventricular overload and left ventricular underfilling. J Am Coll Cardiol 2008; 51: 750-757.

84 Handoko ML, Lamberts RR, Redout EM, et al. Right ventricular pacing improves right heart function in experimental pulmonary arterial hypertension: a study in the isolated heart. Am J Physiol Heart Circ Physiol 2009; 297: H1752-H1759.

85 Dubin AM, Feinstein JA, Reddy VM, et al. Electrical resynchronization: a novel therapy for the failing right ventricle. Circulation 2003; 107: 2287-2289.

86 Hardziyenka M. Interventricular resynchronization therapy in right ventricular failure: a proof-of-concept study in patients with chronic thromboembolic pulmonary hypertension. PhD thesis. University of Amsterdam, Amsterdam, the Netherlands. 2009.

87 Peacock AJ, Naeije R, Galie N, et al. End-points and clinical trial design in pulmonary arterial hypertension: have we made progress? Eur Respir J 2009; 34: 231-242. 